\title{
High energy directly pumped Ho:YLF laser
}

\author{
Mulugeta Petros \\ Science and Technology Corporation, 101 Research Drive, Hampton, VA 23666-1340 \\ e-mail:m.petros@larc.nasa.gov
}

Jirong Yu, Upendra N. Singh and Norman P. Barnes

NASA Langley Research Center MS 474 Hampton, VA 23681-0001

\begin{abstract}
The most commonly used crystal architecture to produce $2 \mu \mathrm{m}$ laser is codoping Ho and Tm into a single host crystal. In this method, the stored energy transfer from the $\mathrm{Tm}^{3} \mathrm{~F}_{4}$ to the $\mathrm{Ho}^{5} \mathrm{I}_{7}$ manifold is not fast enough to warrant high efficiency for short pulse applications. By separating the Ho and the Tm ions and doping the Tm in $\mathrm{YALO}_{3}$ and the Ho in YLF, we were able to directly pump the Ho ${ }^{5} \mathrm{I}_{7}$ manifold with $1.94 \mu \mathrm{m}$. The Ho:YLF laser has produced $33 \mathrm{~mJ}$ at $2.062 \mu \mathrm{m}$ with a quantum efficiency of 0.88 . The performance of each laser will be presented. (C)1999 Optical Society of America

OCIS Codes: (140.0140) Lasers and laser optics; (140.5680) Rare earth and transition metal solid-state lasers.
\end{abstract}

\section{Introduction}

A high-energy two-micron laser has many applications in the area of remote sensing and pollutant detection. Using a co-doped Ho:Tm:YLF crystal, NASA Langley researchers have demonstrated up to $600 \mathrm{~mJ}$ of $2 \mu \mathrm{m}$ energy [1]. Although this laser produced high energy, further efficiency enhancement is necessary if this system is to be considered for a space borne system. In an effort to enhance the efficiency of the $2 \mu \mathrm{m}$-laser system, separating Ho and Tm ions on different individual hosts is actively pursued. The primary advantage of directly pumping the Ho ${ }^{5} \mathrm{I}_{7}$ is that it does not have to depend on energy transfer, which lends itself to various radiative and non-radiative losses [2]. Up-conversion losses that have deleterious effect in high-energy Q-switched lasers are eliminated. In addition, eliminating Ho:Tm energy sharing enhances single Q-switched pulse energy extraction. In recent years, researchers have demonstrated high efficiency in separately doped Tm and Ho crystals [3]. However, most of the work done to date concentrates on $\mathrm{CW}$ pumped repetitively Q-switched lasers. Although some of these lasers have high average power, their energy per pulse is less than $5 \mathrm{~mJ}$. Pulsed high energy is more challenging because fluorescence amplified spontaneous emission and up-conversions all detract from efficiency and optical transparency has to be achieved for every shot. This work concentrates on high energy pulsed laser system which uses $\mathrm{Tm}: \mathrm{YAlO}_{3}$ laser operating at $1.94 \mu \mathrm{m}$ for pumping Ho:YLF to achieve $2.06 \mu \mathrm{m}$ lasing.

\section{Experimental arrangement and results}

In this experiment, Alexandrite laser is used as a pump source for the Tm laser, which we ultimately plan to replace with a diode laser. It is capable of providing up to 2 joules of energy when it is pumped with 143 Joules of electrical energy. The pulse length is extended to $500 \mu$ s to take advantage of the long lifetime of the Tm laser. A birefringent filter is inserted in the resonator to tune the output wavelength around $.795 \mu \mathrm{m}$, which is the peak absorption of the $\mathrm{Tm}: \mathrm{YAlO}_{3}$ laser. The experimental setup is shown in Fig. 1. The beam is then directed with two turning mirrors and focussed on to the $\mathrm{YAlO}_{3}$ crystal. 


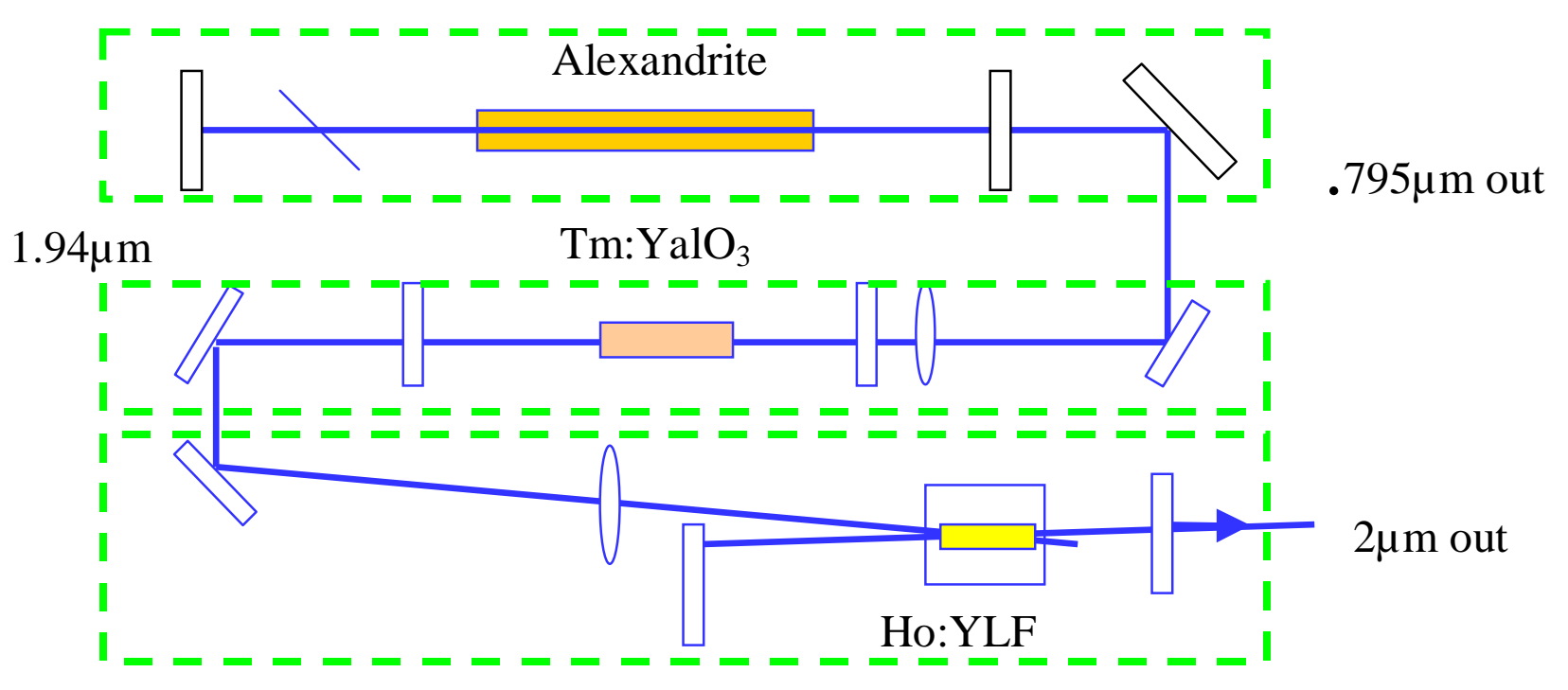

Figure 1. Schematic diagram of $1.94 \mu \mathrm{m}$ pumped Ho:YLF laser

The Tm: $\mathrm{YAlO}_{3}$ crystal is grown and core drilled along the a-axis. It is doped with $.02 \mathrm{Tm}$. The $20 \mathrm{~mm}$ by $4 \mathrm{~mm}$ diameter laser rod is conductive cooled by three cold fingers. The resonator length used for this experiment is 35-centimeters and formed by a curved mirror, which has a 10-meter radius of curvature and flat output couplers. The curved mirror is highly transmissive from $.7 \mu \mathrm{m}$ to $.8 \mu \mathrm{m}$ and highly reflective between $1.9 \mu \mathrm{m}$ and $2.1 \mu \mathrm{m}$.

The Tm laser performed with several output mirrors having transmissions ranging from .05 to .3. When a .05 transmissive mirror is used, the threshold was slightly lower and the output wavelength was around $1.99 \mu \mathrm{m}$. However, this wavelength is out of the absorption range of the Ho:YLF. Such a low transmission mirror also leaves a high circulating fluence inside the resonator, which could potentially, cause optical damage. By using a .28 transmission mirror the internal circulating fluence is reduced from $260 \mathrm{~J} / \mathrm{cm}^{2}$ down to $50 \mathrm{~J} / \mathrm{cm}^{2}$. The output wavelength is $1.94 \mu \mathrm{m}$ without inter-cavity tuning optics, which matches the absorption peak of the Ho: YLF crystal.

The pump polarization is aligned to the b-axis of the crystal to match to the absorption peak. At maximum pump energy, the Tm crystal absorbed over $92 \%$ of the pump energy. The pump beam is focused to match the $\mathrm{TEM}_{\mathrm{oo}}$ mode volume in the Tm rod. Over $240 \mathrm{~mJ}$ of energy at $1.94 \mu \mathrm{m}$ is produced with 1 joule of pump, which represents an optical to optical conversion of $24 \%$ to the TEMoomode. Fig. 2 shows the input output performance of the Tm laser. There is no sign of saturation in the plot, which shows that the laser can produce even higher energy. The output is $300 \mu$ s long highly spiky relaxation oscillation, which is used to pump the Ho laser.

The Ho:YLF resonator consisted of a 30-centimeter radius of curvature mirror and flat output coupler with $13 \%$ transmission. The resonator length is $28 \mathrm{~cm}$, which produced a $250 \mu \mathrm{m} \mathrm{TEM}_{00}$ beam radius at the crystal position. In this experiment, the angle between the pump and the Ho cavity was 4 degrees. The overlap efficiency between the pump and the mode volume is estimated to be around $60 \%$. In future, a dichroic mirror will be used to pump collinearly and thus optimize the spatial overlap of the pump and mode volume. 


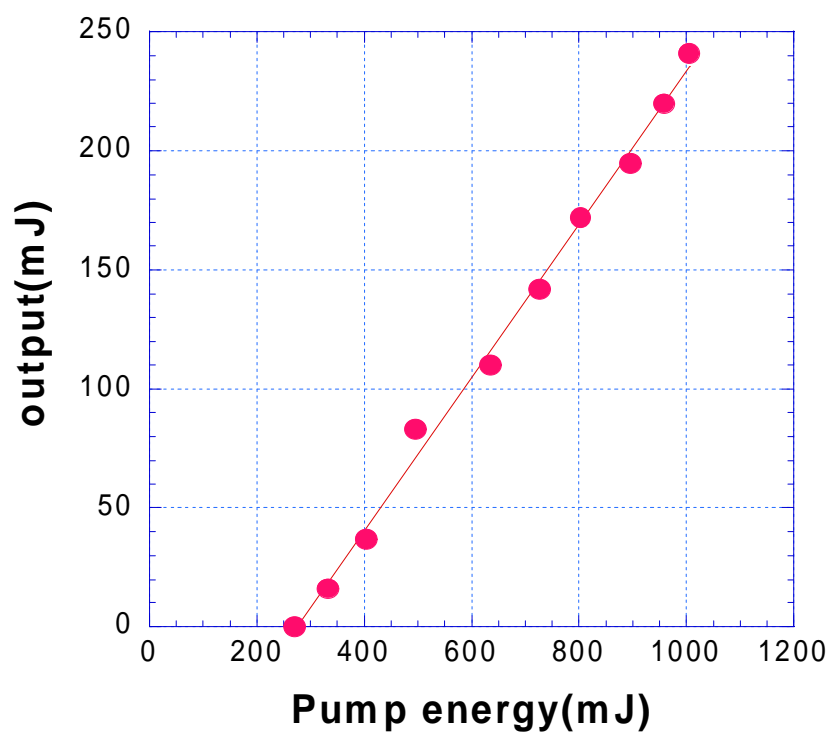

Figure 2. $\mathrm{Tm} \mathrm{YAlO}_{3}$ laser performance of the

A $5 \times 5 \times 10 \mathrm{~mm}$ long and 5x5x5 mm cube Ho:YLF crystals doped with $2 \%$ Ho were evaluated. The crystals were encapsulated in a cooling chamber and the temperature is kept at $-30^{\circ} \mathrm{C}$ to depopulate the Ho lower laser level. We evaluated the temperature effect of this laser by varying the temperature of the $5 \mathrm{~mm}$ cube crystal holder from $-30^{\circ} \mathrm{C}$ to over $60^{\circ} \mathrm{C}$. Laser operation was achieved even at the elevated temperature of more than $61^{\circ} \mathrm{C}$.

The pump beam is focused down to a $500-\mu \mathrm{m}$ waist, which yields $36 \mathrm{~J} / \mathrm{cm}^{2}$ pump fluence at the maximum pump energy. The pump polarization is oriented parallel to the c-axis of the Ho crystal. The absorption efficiency of both crystals is shown in fig.3. At the maximum pump fluence, the absorption is .2 and .3 for the $5 \mathrm{~mm}$ and $10 \mathrm{~mm}$ crystals respectively. It is clear from this plot that the Ho crystal will have to be optimized to absorb more of the pump. This can be achieved by either raising the doping concentration or lengthening the crystal. Care should be exercised not to reach the limit of Ho to Ho upconversion by doping too much Ho. Lengthening the crystal length requires proper focusing optics to match the laser and the pump mode in the resonator. The later is a benign solution since it only deals with geometric problem.

Despite the low absorption, the Ho:YLF laser produced $33 \mathrm{~mJ}$ at $2.062 \mu \mathrm{m}$ for an absorbed energy of only $40 \mathrm{~mJ}$. This equates to a quantum efficiency of 0.88 . In this case quantum efficiency is defined as the ratio of output photons to the absorbed photons. Thus beam overlap, resonator losses and extraction efficiency are all included. The threshold fluence is in the order of 5Joule/ $\mathrm{cm}^{2}$. Fig.4 compares the performance of the two different Ho:YLF crystals.

The output wavelength at low transmission mirrors is $2.062 \mu \mathrm{m}$ and with $30 \%$ output coupler the lasing wavelength changed to $2.05 \mu \mathrm{m}$. This is consistent with the theory that at low loss condition, the transition with a better thermal occupation factor lases while if the loss is higher, the transition with a higher gain is favored.

For coherent lidar applications, it is essential to have diffraction limited beam quality. In this configuration, the thermal loading on the crystal is small. This, coupled with small thermal lensing of YLF, should promote good beam quality with high energy. 


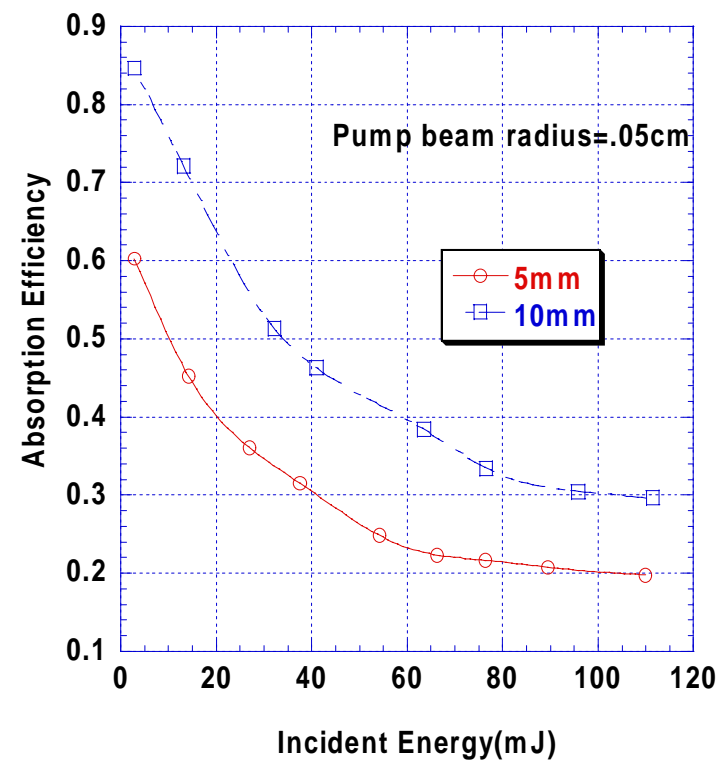

Figure 3. Ho:YLF absorption.

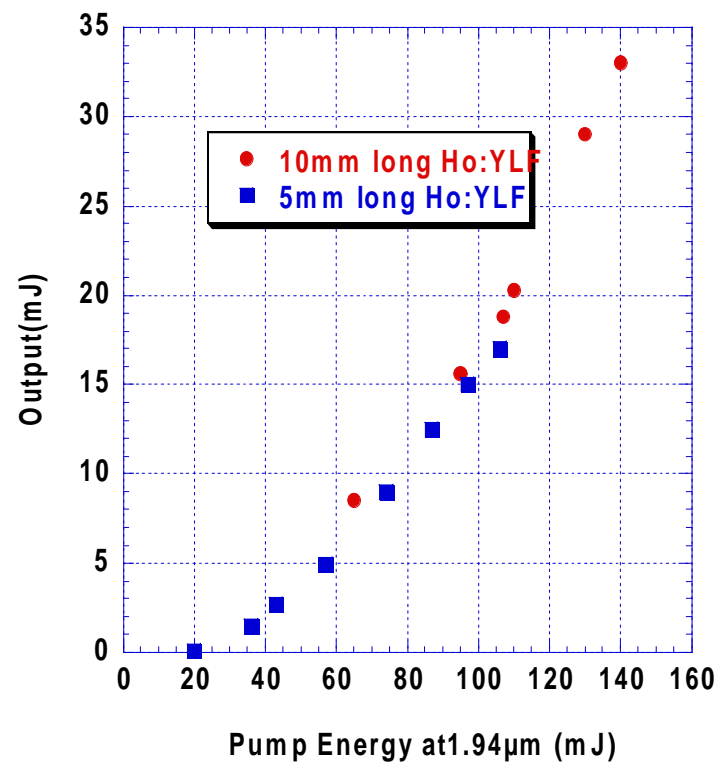

Figure 4. Ho: YLF laser performance

This is an ongoing effort and these are preliminary results that are yet to be optimized. Areas for improving the efficiency have been identified. It is clear from Fig. 3 that the Ho atoms are saturated in both crystals. A higher Ho concentration or a longer crystal can enhance the absorption and hence improve the performance.

\section{Conclusions}

We have demonstrated a Ho:YLF laser with quantum efficiency of 0.88 and energy of $33 \mathrm{~mJ}$. To our knowledge this is the highest energy to date achieved with directly pumped Ho laser. We expect the conversion efficiency of the normal mode to Q-switch mode will be much better than the $30 \%$ commonly achieved in high-energy co-doped Ho:Tm laser systems.

\section{References}

1. Upendra N. Singh, Jirong Yu, Mulugeta Petros, Norman P. Barnes, Julie A. Williams-Byrd, George E Lockard, and Edward A. Modlin "Injection seeded, room temperature, diode pumped Ho:Tm:YLF laser with output energy of 600mJ at $10 \mathrm{~Hz}$." OSA TOPS Advanced Solid State Lasers, Trends in Optics and Photonics, Volume XIX, pp. 194-196, 1998.

2. David W. Hart, Mahendra Jani, Norman P. Barnes "Room-temperature lasing of End pumped $\mathrm{Ho}_{\mathrm{L}} \mathrm{Lu}_{3} \mathrm{Al}_{5} \mathrm{O}_{12}$ " Opt. Lett., 21, No. 10, pp. 728-730, 1996.

3. P. A. Bundi, L. A. Pomeranz, M. L. Lemons, P. G. Schunemann, T. M. Pollak, J. R. Mosto and E. P. Chiklis "CW and Q-switched Ho:YAG pumped by Tm:YALO" OSA TOPS Advanced Solid State Lasers, Trends in Optics and Photonics, Volume XIX, pp. 204-205, 1998. 